\title{
CENP-C Phosphorylation by CDK1 in vitro
}

Reito Watanabe, Masatoshi Hara, Mariko Ariyoshi and Tatsuo Fukagawa*

Graduate School of Frontier Biosciences, Osaka University, Suita, Osaka 565-0871, Japan

*For correspondence: tfukagawa@fbs.osaka-u.ac.jp

[Abstract] Accurate chromosome segregation during mitosis requires the kinetochore, a large protein complex, which makes a linkage between chromosomes and spindle microtubes. An essential kinetochore component, CENP-C, is phosphorylated by Cyclin-B-Cyclin dependent kinase 1 (CDK1) that is a master kinase for mitotic progression, promoting proper kinetochore assembly during mitosis. Here, we describe an in vitro CDK1 kinase assay to detect CENP-C phosphorylation using Phos-tag SDS-PAGE without radiolabeled ATP. Our protocol has advantages in ease and safety over conventional phosphorylation assays using $\left[\mathrm{Y}^{-}{ }^{32} \mathrm{P}\right]-\mathrm{ATP}$, which has potential hazards despite their better sensitivity. The protocol described here can be applicable to other kinases and be also useful for analysis of phospho-sites in substrates in vitro.

Keywords: In vitro kinase assay, CDK1, Phos-tag, Mitosis, Centromere, Kinetochore

[Background] Cyclin-B-Cyclin dependent kinase 1 (CDK1), which is a master regulator of mitosis, phosphorylates numerous targets to ensure mitotic progression (Nurse, 1990; Malumbres and Barbacid, 2005). During mitosis, chromosomes carrying the genetic information are equally divided into two daughter cells. The kinetochore is a key large protein complex ensuring the faithful chromosome segregation by bridging between chromosomes and spindle microtubules (Fukagawa and Earnshaw, 2014). Various proteins composing the kinetochore are phosphorylated by CDK1 (Gascoigne et al., 2013; Nishino et al., 2013; Hara et al., 2018b; Watanabe et al., 2019). The CDK1 phosphorylation plays critical roles in kinetochore assembly (Gascoigne et al., 2013) and also in the correct microtubule attachment (Nishino et al., 2013; Hara et al., 2018a).

In vitro kinase assays and phospho-protein analyses are important ways, which provide us understanding of how phospho-regulations are achieved in kinetochore assembly and function. A classical way to detect phospho-proteins is a radiolabeled assay using [Y- $\left.{ }^{32} \mathrm{P}\right]-A T P$. However, although the radiolabeled assay gives high sensitivity, it has potential hazards. To use radioactivity, we would need to take a radiation safety training course, and prepare controlled area and safety protection equipment. Phospho-specific antibodies can be alternative ways. However, the antibodies are not always available for targets of interest.

Here, we describe a method to phosphorylate a kinetochore protein, CENP-C, by CDK1 in vitro and to detect the phosphorylated CENP-C using Phos-tag SDS-PAGE (Kinoshita et al., 2006). Phos-tag is 1,3-bis[bis(pyridin-2-ylmethyl)amino]propan-2-olato dizinc(II) complex that has a vacancy on two metal ions and binds to phenyl phosphate dianion on the target protein via two metal ions. Phospho-proteins migrate slower in the SDS-PAGE containing Phos-tag. This method magnifies mobility shifts of the 
phospho-proteins, which even show no migration changes in conventional SDS-PAGE. The protocol can be applicable to detect phospho-proteins by other kinases. Given that mobility of the phosphoprotein on Phos-tag SDS-PAGE changes with number of phosphorylation-sites as well as location of the phosphorylation-sites, the procedure could be also utilized for analysis of phosphorylation-sites. The protocol is easy and safe, and can be finished within a few hours including kinase reaction and detection, saving time compared with the conventional radiolabeled assays.

\section{Materials and Reagents}

1. Active cyclin B-CDK1 purified as described previously (Okumura et al., 1996; Watanabe et al., 2019)

2. Recombinant chicken MBP (maltose-binding protein)-CENP-C (aa 601-864) purified as described previously (Watanabe et al., 2019)

3. Xpress Micro Dialyzer MD 100 (Scienova, catalog number: 40075)

4. Protein Lobind Tube $500 \mu \mathrm{l}$ (Eppendorf, catalog number: 0030108116)

5. Super Sep ${ }^{\mathrm{TM}}$ Ace, 5-20\%, 17-well (FUJIFILM Wako, catalog number: 194-15021)

6. CBB Stain One (Nacalai Tesque, catalog number: 04543-51)

7. cOmplete ${ }^{\mathrm{TM}}$ EDTA-free proteinase inhibitor (Roche, catalog number: 11873580001))

8. Phos-tag Acrylamide (FUJIFILM Wako, catalog number: 300-93523) (Kinoshita et al., 2006)

9. Tris (Trizma ${ }^{\circledR}$ base) (Sigma-Aldrich, catalog number: T1503-1KG)

10. $\mathrm{MgCl}_{2}$ (Nacalai Tesque, catalog number: 20909-55)

11. $\mathrm{NaCl}$ (Nacalai Tesque, catalog number: $31320-05$ )

12. EDTA (Ethylenediaminetetraacetic acid, Nacalai Tesque, catalog number: 15111-45)

13. $\mathrm{HCl}$ (Nacalai Tesque, catalog number: 18321-05)

14. $\mathrm{NaOH}$ (Nacalai Tesque, catalog number: $31511-05$ )

15. 30(w/v)\%-Acrylamide/Bis Mixed Solution (29:1) (Nacalai Tesque, catalog number: 06141-35)

16. ATP (Adenosine triphosphate) (Nacalai Tesque, catalog number: 01072-11)

17. SDS (Sodium dodecyl sulfate) (Nacalai Tesque, catalog number: 02873-75)

18. APS (Ammonium peroxodisulfate) (Nacalai Tesque, catalog number: 02627-34)

19. TEMED ( $N, N, N^{\prime}, N^{\prime}-$ Tetramethylethylenediamine) (Nacalai Tesque, catalog number: 33401-72)

20. $\mathrm{MnCl}_{2}$ (FUJIFILM Wako, catalog number: 133-00725)

21. Glycerol (Nacalai Tesque, catalog number: 17018-83)

22. 2-mercaptoethanol (SIGMA, catalog number: M3148-100ML)

23. Bromophenol blue (FUJIFILM Wako, catalog number: 101123)

24. Glycine (Nacalai Tesque, catalog number: 17109-35)

25. Precision Plus Protein Dual Color standards (Bio-Rad, catalog number: 161-0374)

26. $1 x$ kinase buffer (KB) (see Recipes)

27. $2 x$ kinase buffer (see Recipes)

28. $25 x$ cOmplete ${ }^{\mathrm{TM}}$ EDTA-free proteinase inhibitor (see Recipes) 
Please cite this article as: Watanabe et. al., (2021). CENP-C Phosphorylation by CDK1 in vitro,Bio-protocol 11 (1): e3879. DOI: 10.21769/BioProtoc.3879.

29. Phos-tag 5(w/v)\%-Acrylamide/Bis (29:1) gel (see Recipes)

Separation gel

Stacking gel

30. SDS-PAGE running buffer (see Recipes)

31. $2 x$ Laemmli sample buffer (see Recipes)

32. 1x Laemmli sample buffer (see Recipes)

\section{Equipment}

1. NanoDrop 2000c Spectrometer (Thermo Fisher Scientific, model: NanoDrop ${ }^{\mathrm{TM}} 2000 \mathrm{C}$ Spectrophotometer, catalog number: ND2000C)

2. SDS-PAGE gel electrophoresis chamber (BIO CRAFT, catalog number: BE-230G)

3. Scanner (EPSON, model: GT-X980)

4. Invitroshaker (TAITEC, model: Shake-LR, catalog number: 0054809-000)

\section{Software}

1. ImageJ 1.8.0_172 (Abramoff et al., 2004)

\section{Procedure}

A. Preparation of MBP-CENP-C (aa 601-864)

Note: Although we describe the protocol for MBP-CENP-C (aa 601-864) phosphorylation using $C D K 1$, the protocol can be applied for other kinases and their substrates for your interests.

1. Purify recombinant MBP-CENP-C (aa 601-864) as described in Watanabe et al. (2019).

Note: Briefly, the MBP-CENP-C (aa601-864) was expressed in bacteria, and purified with Amylose resin and Hi-Trap SP column with Superdex 200 pg. The purified protein was dissolved with buffer containing $20 \mathrm{mM}$ HEPES-NaOH (pH 7.5), $500 \mathrm{mM} \mathrm{NaCl}, 5 \%$ Glycerol, 1 mM EDTA$\mathrm{NaOH}$ ( $\mathrm{pH}$ 8.0), and $1 \mathrm{mM} D T T$. The purified protein was snap-frozen in liquid nitrogen and stored at $-80^{\circ} \mathrm{C}$. The frozen protein should be thawed on ice just before using.

2. Prepare $1,000 \mathrm{ml}$ of ice-cold $1 \mathrm{x}$ kinase buffer (listed in Recipes).

3. Inject MBP-CENP-C (aa 601-864) into Xpress Micro Dialyzer up to $100 \mu$ following the product instruction manuals and dialyze the protein in $1,000 \mathrm{ml}$ of $1 \mathrm{x}$ kinase buffer for overnight at $4{ }^{\circ} \mathrm{C}$. Note: Dialysis is important for removal of EDTA from your protein samples, and for adjustment of salt concentration for the next steps. Because high concentration of salt and EDTA inhibits kinase reaction and interferes phospho-protein running on Phos-tag SDS-PAGE.

4. Collect the dialyzed MBP-CENP-C (aa 601-864) into a Protein Lobind Tube on ice.

5. Analyze protein concentration using molecular absorptivity and molecular weight with NanoDrop 2000c Spectrometer (Desjardins et al., 2009). 
Note: Because in the next step the substrate is added for kinase reaction at $2 \mathrm{mg} / \mathrm{ml}$ (final concentration), if concentration of the protein is less than $2 \mathrm{mg} / \mathrm{ml}$, concentrate protein using Amicon Ultra.

B. Optimization of kinase reaction

Note: To get optimum phosphorylation in the kinase reaction, several conditions can be optimized: substrate concentration, kinase amount, reaction buffer, and reaction time and temperature. Here, we demonstrate an example of optimization for reaction time in the kinase assay.

1. Mix the dialyzed MBP-CENP-C (aa 601-864) in kinase buffer with 100 mM ATP, 25x cOmplete ${ }^{T M}$ EDTA-free proteinase inhibitor (listed in Recipes), active cyclin B-CDK1 (relative H1 kinase activity: $1.4 \mathrm{pmol} \mathrm{P} / \mathrm{min} \mu \mathrm{l}$ ), and add $2 \mathrm{x}$ kinase buffer (listed in Recipes) and $\mathrm{H}_{2} \mathrm{O}$ to adjust concentration of each component to final concentration in $1 \times$ kinase buffer as described in Table 1 showing our representative $10 \mu$ l-scale reaction mixture.

Notes:

a. You can change the volume of the kinase reaction according to your experimental design.

b. It is recommended to make small aliquots and store $100 \mathrm{mM}$ ATP at $-20^{\circ} \mathrm{C}$ to avoid multiple freeze-thaw cycles.

c. Purification of active cyclin B-CDK1 was described in Okumura et al. (1996).

Table 1. Representative kinase reaction mix

\begin{tabular}{|c|c|c|c|}
\hline & Stock concentration & Volume $(\mu \mathrm{l})$ & Final concentration \\
\hline MBP-CENP-C (aa 601-864) in x1 KB & $4 \mathrm{mg} / \mathrm{ml}$ & 5 & $2 \mathrm{mg} / \mathrm{ml}$ \\
\hline ATP & $100 \mathrm{mM}$ & 0.1 & $2 \mathrm{mM}$ \\
\hline cOmplete $^{T M}$ EDTA-free proteinase & $x 25$ & 0.4 & $\mathrm{x} 1$ \\
\hline \multicolumn{4}{|l|}{ inhibitor } \\
\hline Active cyclin B-CDK1 & $1.4 \mathrm{pmol} \mathrm{P} / \mathrm{min} \mu \mathrm{l}$ & 0.1 & $0.014 \mathrm{pmol} \mathrm{P} / \mathrm{min} \mu \mathrm{l}$ \\
\hline Kinase buffer (KB) & $x 2$ & 2.5 & $\mathrm{x} 1$ \\
\hline $\mathrm{H}_{2} \mathrm{O}$ & & 1.9 & \\
\hline Total & & 10 & \\
\hline
\end{tabular}

2. Incubate the $10 \mu \mathrm{l}$ reaction mixture at $25^{\circ} \mathrm{C}$. Gently mix the sample with tapping every $15 \mathrm{~min}$. Note: In this protocol, to examine optimum reaction time, several $10 \mu$-scale reactions are incubated for the indicated time periods in Figure 1 (0, 30, 60 and $90 \mathrm{~min}$ ).

3. To stop the reaction, add $10 \mu \mathrm{l}$ of $2 \mathrm{x}$ Laemmli sample buffer (listed in Recipes) to the reaction mixture. Then, add further $20 \mu \mathrm{l}$ of $1 \mathrm{x}$ Laemmli sample buffer to adjust protein concentration for SDS-PAGE in the next step (listed in Recipes), and heat samples for 5 min at $96{ }^{\circ} \mathrm{C}$.

Note: If you use the phosphorylated proteins for other in vitro assays such as a pull-down assay, stop the kinase reaction by adding $1 \mathrm{MEDTA}-\mathrm{NaOH}(\mathrm{pH}$ 8.0) to the reaction mixture in a final concentration of $5 \mathrm{mM}$. 
C. Detection of phosphorylated substrates using Phos-tag SDS-PAGE

1. Prepare a Phos-tag 5(w/v)\%-Acrylamide/Bis (29:1) gel (25 $\mu \mathrm{M}$ Phos-tag, listed in Recipes). Note: To obtain optimum separation of phosphorylated proteins in the Phos-tag SDS-PAGE, Phos-tag and acrylamide concentrations should be optimized. Assemble the gel into an electrophoresis chamber (listed in Equipment).

2. Add SDS-PAGE running buffer to electrophoresis chamber (listed in Recipes).

3. Load $0.5 \mu \mathrm{g}$ of phosphorylated protein each lane in $1 \mathrm{x}$ Laemmli sample buffer, and also $5 \mu \mathrm{l}$ of molecular weight marker on the gel.

Note: If your molecular weight marker includes salts and/or EDTA, which interfere sample running in the next lanes on Phos-tag SDS-PAGE. To avoid this problem, an empty lane should be inserted between the molecular weight marker and the next sample.

4. Run gels using $35 \mathrm{~mA}$ constant current for $55 \mathrm{~min}$.

5. After electrophoresis, wash gels with water for 5 min using Invitroshaker (speed 60).

6. Remove water and fill gel with $50 \mathrm{ml}$ of CBB Stain One. Warm the gel with CBB stain One by a $700 \mathrm{~W}$ microwave oven for $1 \mathrm{~min}$ (approximately $70^{\circ} \mathrm{C}$ ).

7. Shake the gel for $10 \mathrm{~min}$ at room temperature using Invitroshaker (speed 60).

8. Remove CBB stain One and wash gel with water for 1 min twice using Invitroshaker (speed 60).

9. Fill gel with $50 \mathrm{ml}$ of new water. Warm the gel with water by a $700 \mathrm{~W}$ microwave oven for $1 \mathrm{~min}$ (approximately $70^{\circ} \mathrm{C}$ ).

10. Shake the gel with warmed water over $60 \mathrm{~min}$ at room temperature using Invitroshaker (speed $60)$.

11. Remove water and wash gel with water twice and acquire images of the stained gel using an image scanner.

D. Detection of phosphorylated substrates using regular SDS-PAGE

Note: To examine protein levels and qualities of substrates, use a conventional SDS-PAGE, because it is difficult to compare protein levels precisely and detect small amount of protein fragments on the Phos-tag SDS-PAGE. This procedure is optional, but we highly recommend it, because some proteins may be degraded during the kinase reaction.

1. Prepare Super Sep ${ }^{\mathrm{TM}}$ Ace, 5-20\%, 17-well SDS precast gel.

2. Assemble the gel into an electrophoresis chamber (listed in Equipment).

3. Add SDS-PAGE running buffer to electrophoresis chamber (listed in Recipes).

4. Load $0.5 \mu \mathrm{g}$ of phosphorylated protein each lane in $1 \mathrm{x}$ Laemmli sample buffer, and also $5 \mu \mathrm{l}$ of molecular weight marker on the gel.

5. Run gels using $35 \mathrm{~mA}$ constant current for $55 \mathrm{~min}$.

6. After electrophoresis, wash gels with water for $5 \mathrm{~min}$.

7. Remove water and fill gel with $50 \mathrm{ml}$ of CBB Stain One. Warm the gel with CBB stain One by a $700 \mathrm{~W}$ microwave oven for $1 \mathrm{~min}$ (approximately $70^{\circ} \mathrm{C}$ ).

8. Shake the gel for $10 \mathrm{~min}$ at room temperature using Invitroshaker (speed 60). 
9. Remove $\mathrm{CBB}$ stain One and wash gel with water for 1 min twice using Invitroshaker (speed $60)$.

10. Fill gel with $50 \mathrm{ml}$ of new water. Warm the gel with water by a $700 \mathrm{~W}$ microwave oven for $1 \mathrm{~min}$ (approximately $70^{\circ} \mathrm{C}$ ).

11. Shake the gel with warmed water over $60 \mathrm{~min}$ at room temperature using Invitroshaker (speed $60)$.

12. Remove water and wash gel with water twice and acquire images of the stained gel using an image scanner.

\section{Data analysis}

In Phos-tag SDS-PAGE, if protein bands are shifted up in the kinase-treated sample compared with the untreated sample protein band, it indicates that the substrate is phosphorylated by kinase in vitro. The band shift in Phos-tag SDS-PAGE depends on the number of phosphorylated residues and their position in the substrate.

Figure 1 shows representative images of phosphor-protein analysis on Phos-tag (left) and conventional (right) SDS-PAGE gels using the protocol described in this chapter. The MBP-CENPC (aa 601-864) treated with CDK1 clearly shows mobility shifts on Phos-tag SDS-PAGE, indicating CENP-C phosphorylation by CDK1. Importantly, the Phos-tag SDS-PAGE enable to detect the mobility shifts of phosphor-proteins, which do not change their mobility on the conventional SDSPAGE. Phosphorylation levels of the substrate can be evaluated by quantification of the intensity of the shifted bands using ImageJ open source software (Abramoff et al., 2004).

At 30 min after CDK1 treatment, MBP-CENP-C (aa 601-864) displayed an obvious shifted band with reduced levels of unphosphorylated protein band (Figure 1, left). Subsequently, at the later time points, MBP-CENP-C (aa 601-864) showed further mobility shift, suggesting that MBP-CENP-C (aa 601-864) was phosphorylated at two sites by CDK1, but CDK1 appeared to have different phosphorylation efficiency between the two sites.

In summary, this in vitro kinase assay protocol using Phos-tag SDS-PAGE is a simple but useful method to detect kinase activity and to analyze protein phosphorylation. 
A
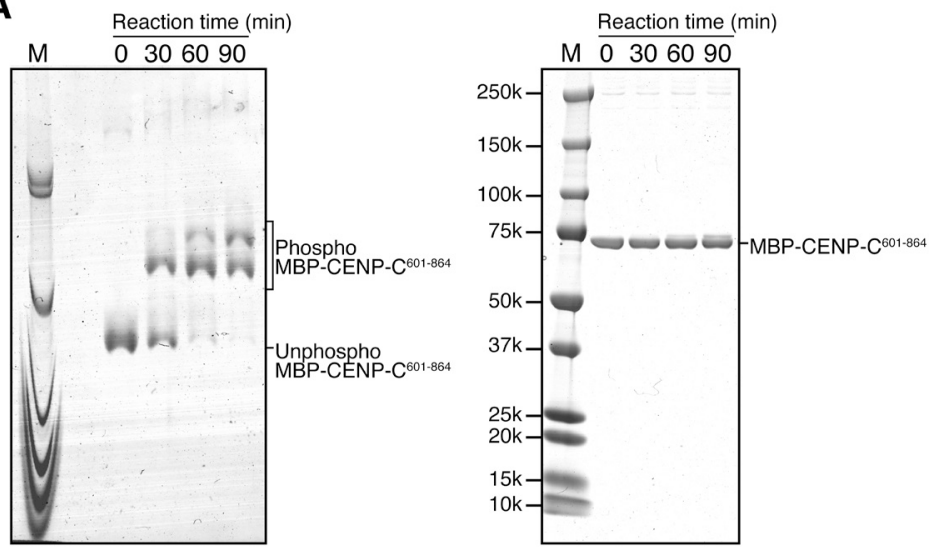

B

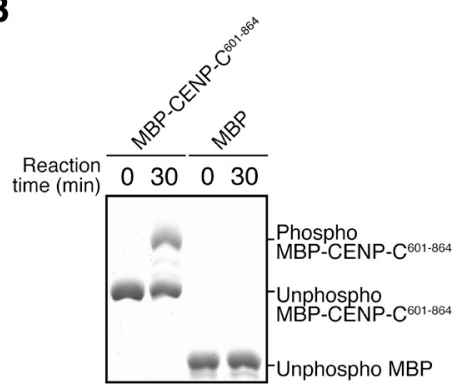

Figure 1. Analysis of CDK1-mediated CENP-C phosphorylation on Phos-tag and conventional SDS-PAGE. A. The left image shows Phos-tag SDS-PAGE analysis of MBP (maltose-binding protein)-CENP-C (aa 601-864): MBP-CENP-C ${ }^{601-864}$ was treated with CDK1 for the indicated times. Right image shows conventional SDS-PAGE analysis of same samples. $M$ shows molecular weight marker. In the left Phos-tag SDS-PAGE analysis, MBP-CENP-C ${ }^{601-}$ ${ }^{864}$ was shifted-up, indicating CENP-C phosphorylation by CDK1 in vitro. B. The image shows Phos-tag SDS-PAGE analysis of MBP-CENP-C ${ }^{601-864}$ and MBP, which were treated with CDK1 for the indicated times. MBP, which is not a CDK1 substrate, showed no mobility shifts, indicating that phosphorylated proteins can be specifically detected on Phos-tag SDS-PAGE.

\section{Recipes}

1. $1 \mathrm{x}$ kinase buffer (KB)

$10 \mathrm{mM}$ Tris-HCl, $\mathrm{pH} 7.5$

$2 \mathrm{mM} \mathrm{MgCl} 2$

$150 \mathrm{mM} \mathrm{NaCl}$

2. $2 x$ kinase buffer

$20 \mathrm{mM}$ Tris- $\mathrm{HCl}, \mathrm{pH} 7.5$

$4 \mathrm{mM} \mathrm{MgCl}_{2}$

$300 \mathrm{mM} \mathrm{NaCl}$

3. $25 \mathrm{x}$ cOmplete ${ }^{\mathrm{TM}}$ EDTA-free proteinase inhibitor 
One tablet of cOmplete ${ }^{\mathrm{TM}}$ EDTA-free proteinase inhibitor in $2 \mathrm{ml}$ water

4. Phos-tag $5(\mathrm{w} / \mathrm{v}) \%$-Acrylamide/Bis $(29: 1)$ gel

\section{Separation gel}

$5(\mathrm{w} / \mathrm{v}) \%$-Acrylamide/Bis Mixed Solution (29:1)

$375 \mathrm{mM}$ Tris- $\mathrm{HCl}, \mathrm{pH} 8.8$

$0.1 \%$ SDS

$0.1 \%$ APS

$0.1 \%$ TEMED

$25 \mu \mathrm{M}$ Phos-tag Acrylamide

$50 \mu \mathrm{M} \mathrm{MnCl} 2$

Note: It is recommended to make small aliquots and store $100 \mu \mathrm{M} \mathrm{MnCl}_{2}$ at $-20{ }^{\circ} \mathrm{C}$ to avoid multiple freeze-thaw cycles. Use of an old $\mathrm{MnCl}_{2}$ stock would cause inefficient mobility changes of phospho-proteins on Phos-tag SDS-PAGE.

\section{Stacking gel}

3.5(w/v)\%-Acrylamide/Bis Mixed Solution (29:1)

$120 \mathrm{mM}$ Tris- $\mathrm{HCl}, \mathrm{pH} 6.8$

$0.1 \%$ SDS

$0.2 \%$ APS

$0.2 \%$ TEMED

5. SDS-PAGE running buffer

$25 \mathrm{mM}$ Tris

192 mM Glycine

$0.1 \%$ SDS

6. $2 x$ Laemmli sample buffer

$0.125 \mathrm{M}$ Tris- $\mathrm{HCl}, \mathrm{pH} 6.8$

$20 \%$ glycerol

$4 \%$ SDS

10\% 2-mercaptoethanol

$0.005 \%$ bromophenol blue

7. $1 \mathrm{x}$ Laemmli sample buffer

0.0625 M Tris-HCl, pH 6.8

$10 \%$ glycerol

$2 \%$ SDS

5\% 2-mercaptoethanol

$0.0025 \%$ bromophenol blue 
Please cite this article as: Watanabe et. al., (2021). CENP-C Phosphorylation by CDK1 in vitro,Bio-protocol 11 (1): e3879. DOI: $10.21769 / B i o P r o t o c .3879$.

\section{Acknowledgments}

This work was supported by JSPS KAKENHI Grant Number 17H06167, $16 \mathrm{H} 06279$ and $15 \mathrm{H} 05972$ to TF, JSPS KAKENHI Grant Number 16K18491 to MH, and JSPS KAKENHI Grant Number $18 \mathrm{~K} 06084$ to MA. This protocol was adapted from and used in Watanabe et al. (2019).

\section{Competing interests}

The authors declare no competing interests.

\section{References}

1. Abramoff, M. D., Magalhães, P. J. and Ram, S. J. (2004). Image processing with ImageJ. Biophotonics Int 11(7): 36-42

2. Desjardins, P., Hansen, J. B. and Allen, M. (2009). Microvolume protein concentration determination using the NanoDrop 2000c spectrophotometer. J Vis Exp (33): e1610.

3. Fukagawa, T. and Earnshaw, W. C. (2014). The centromere: chromatin foundation for the kinetochore machinery. Dev Cell 30(5): 496-508.

4. Gascoigne, K. E. and Cheeseman, I. M. (2013). CDK-dependent phosphorylation and nuclear exclusion coordinately control kinetochore assembly state. J Cell Biol 201(1): 23-32.

5. Hara, M., Ariyoshi, M., Okumura, E. I., Hori, T. and Fukagawa, T. (2018a). Multiple phosphorylations control recruitment of the KMN network onto kinetochores. Nat Cell Biol 20(12): 1378-1388.

6. Hara, M. and Fukagawa, T. (2018b). Kinetochore assembly and disassembly during mitotic entry and exit. Curr Opin Cell Biol 52: 73-81.

7. Kinoshita, E., Kinoshita-Kikuta, E., Takiyama, K. and Koike, T. (2006). Phosphate-binding tag, a new tool to visualize phosphorylated proteins. Mol Cell Proteomics 5(4): 749-757.

8. Nishino, T., Rago, F., Hori, T., Tomii, K., Cheeseman, I. M. and Fukagawa, T. (2013). CENP-T provides a structural platform for outer kinetochore assembly. EMBO J 32(3): 424-436.

9. Nurse, P. (1990). Universal control mechanism regulating onset of M-phase. Nature 344(6266): 503-508.

10. Malumbres, M. and Barbacid, M. (2005). Mammalian cyclin-dependent kinases. Trends Biochem Sci 30(11): 630-641.

11. Okumura, E., Sekiai, T., Hisanaga, S., Tachibana, K. and Kishimoto, T. (1996). Initial triggering of M-phase in starfish oocytes: a possible novel component of maturation-promoting factor besides cdc2 kinase. J Cell Biol 132(1-2): 125-135.

12. Watanabe, R., Hara, M., Okumura, E. I., Herve, S., Fachinetti, D., Ariyoshi, M. and Fukagawa, T. (2019). CDK1-mediated CENP-C phosphorylation modulates CENP-A binding and mitotic kinetochore localization. J Cell Biol 218(12): 4042-4062. 